\title{
Fever of undetermined origin in a patient with pyogenic arthritis, pyoderma gangrenosum, and acne (PAPA syndrome)
}

\author{
José Roberto Lambertucci ${ }^{[1]}$
}

[1]. Faculdade de Medicina, Universidade Federal de Minas Gerais, Belo Horizonte, MG
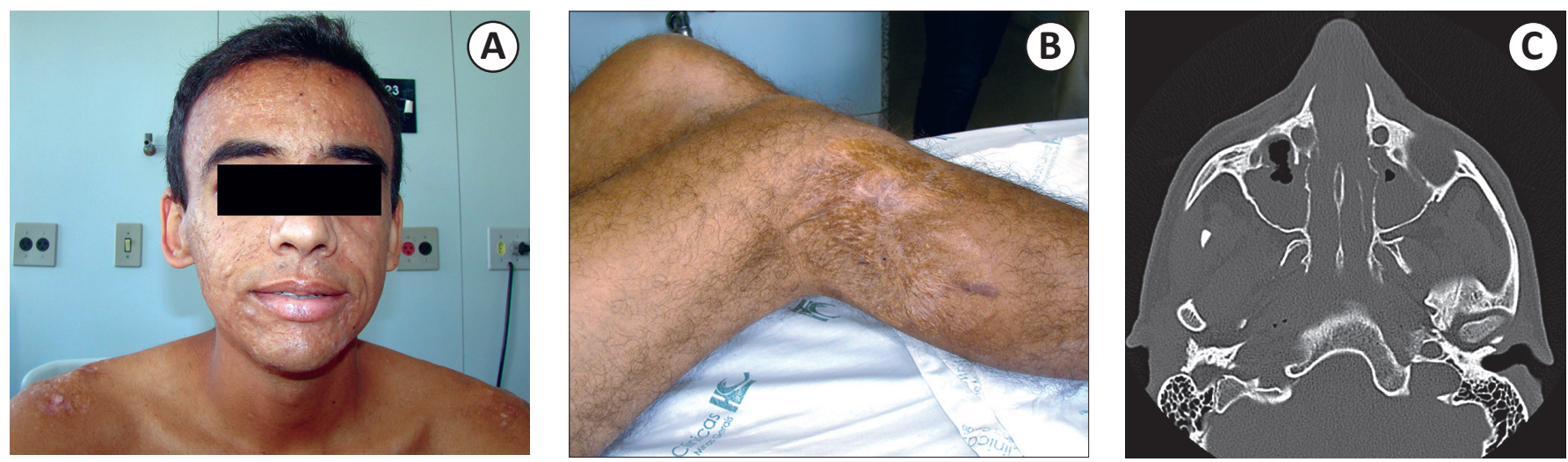

A 22-year-old man was referred to our clinic with a history of recurrent fever, arthalgia, and swelling on the right knee since childhood. At the age of 13, he noted the presence of nodulocystic acne on the face (Figure A). He also developed painful ulcers on both lower legs following a minor trauma and received a skin graft on an extensive ulcer on the right leg (Figure B). In his first admission to our clinic, he had a fever, pancytopenia, and a cough, and a computed tomography (CT) scan showed pansinusitis (Figure C). He was taking prednisone (20 mg/day) and methotrexate. He was administered amoxicillin and clavulanate for 14 days, and the fever resolved. A surgical drainage of the left maxillary sinus was performed, but no bacterium or fungus grew in the culture of the drained sludge. A bone marrow biopsy was compatible with myelodysplastic syndrome. He was followed at the outpatient clinic; however, he eventually developed acute myeloid leukemia and died a few days later in the intensive care unit. Hereditary autoinflammatory syndromes are an emerging group of inflammatory diseases distinct from infectious, autoimmune, and allergic disorders.
The term "autoinflammatory syndrome" describes an autosomal dominant periodic fever caused by mutations in the tumor necrosis factor type 1 receptor. A mutation outside of the CD2BP1 gene that results in an altered interaction between CD2BP1 and pyrin might manifest similarly to the pyogenic arthritis, pyoderma gangrenosum, and acne (PAPA) syndrome.

\section{REFERENCES}

1. Jin-Bon H, Yi-Ning S, Hsien-Ching C. Pyogenic arthritis, pyoderma gangrenosum, and acne syndrome (PAPA syndrome): Report of a sporadic case without an identifiable mutation in the CD2BP1 gene. J Am Acad Dermatol 2008; 61:533-535.

2. Braun-Falco M, Kovnerystyy O, Lohse P, Ruzicka T. Pyoderma gangrenosum, acne, and suppurative hidradenitis (PASH) - a new autoinflammatory syndrome distinct from PAPA syndrome. J Am Acad Dermatol 2012; 66:409-415.

3. Lambertucci JR, Rayes AA, Nunes F, Landazuri-Palacios JE, Nobre V. Fever of undetermined origin in patients with the acquired immunodeficiency syndrome in Brazil: report on 55 cases. Rev Inst Med Trop Sao Paulo 1999; 41:27-32.

Address to: Dr. José Roberto Lambertucci. Depto Clínica Médica/Serviço de Doenças Infecciosas e Parasitárias/UFMG. Av. Alfredo Balena 190, 30130-100 Belo Horizonte, MG, Brasil.

Phone: 5531 3337-7781; Fax: $55313409-9820$

e-mail: lamber@uai.com.br

Received 4 January 2014

Accepted 22 January 2014 